\title{
Migração de informações na perspectiva do modelo Open Archival Information System
}

\author{
Migration of information in perspective of the Open Archival Information System
}

\author{
Henrique Machado dos Santos \\ Mestre em Patrimônio Cultural, Programa de Pós-Graduação Profissional da Universidade Federal de Santa \\ Maria - UFSM, Brasil. \\ Arquivista da Coordenação de Arquivo Geral da Universidade Federal do Rio Grande - FURG, Brasil. \\ E-mail: henrique.hms.br@gmail.com
}

\begin{abstract}
Daniel Flores
Doutor em Metodologías y Líneas de Investigación en Biblioteconomía y Documentación pela Universidad de Salamanca, Espanha. Professor associado do Departamento de Ciência da Informação da Universidade Federal Fluminense - UFF, Brasil. E-mail: dfloresbr@gmail.com
\end{abstract}

\section{Resumo}

Este estudo realiza uma reflexão sobre a implementação das estratégias de migração no âmbito dos repositórios digitais em conformidade com o modelo Open Archival Information System - OAIS. Dessa forma, descreve os procedimentos para garantir a manutenção da autenticidade e o acesso contínuo às informações no longo prazo. A metodologia consiste no levantamento bibliográfico de materiais previamente publicados, composto por livros e artigos científicos recuperados pela ferramenta de pesquisa Google Scholar. O modelo OAIS é utilizado como prisma da discussão, de modo que as estratégias de migração representam uma categoria norteadora deste estudo. A análise dos dados coletados é de natureza qualitativa e a discussão segue a lógica dedutiva, consequentemente, obtém-se um artigo de revisão assistemática. Observa-se que o repositório que segue o OAIS é um ambiente confiável para executar estratégias de migração e registrá-las por meio de metadados para manter a autenticidade e garantir que a comunidade designada tenha acesso contínuo à informação no longo prazo. Portanto, clarifica a relação entre OAIS e as estratégias de migração, logo, visa ampliar a discussão em torno dos repositórios digitais. Esta reflexão fornece subsídio teórico aos pesquisadores recém-iniciados no tema e demonstra que a discussão sobre as estratégias de preservação merece novas releituras, então direcionadas aos repositórios digitais em conformidade com o OAIS.

Palavras-chave: Preservação digital. Estratégias de migração. Repositórios digitais. Documentos digitais. Confiabilidade.

\begin{abstract}
This study makes a reflection on the implementation of migration strategies within the digital repositories in accordance with the model Open Archival Information System - OAIS. In this way, it describes the procedures to guarantee the maintenance of authenticity and the continuous access to the information in long-term. The methodology consists on bibliographical survey of previously published materials, composed of books and scientific articles retrieved by the Google Scholar research tool. The OAIS model is used as prism of discussion, so that migration strategies represent a guiding category of this study. The analysis of collected data is of qualitative nature and the discussion follows the deductive logic, consequently, an article of not systematic review is obtained. It is noted that the repository following the OAIS is a trusted environment for executing migration strategies and registering them through metadata to maintain authenticity and ensure that the designated community has continuous access to information in the long-term. Therefore, it clarifies the relationship between OAIS and migration strategies, and thus aims to broaden the discussion around digital repositories. This reflection provides theoretical support to the researchers newly initiated in subject and demonstrates that discussion about preservation strategies deserves new re-reading, then directed to digital repositories in accordance with OAIS.
\end{abstract}

Keywords: Digital preservation. Migration strategies. Digital repositories. Digital records. Reliability.

InCID: R. Ci. Inf. e Doc., Ribeirão Preto, v. 11, n. 1, p. 213-232, mar./ago. 2020. 


\section{Introdução}

A preservação digital surgiu como uma intervenção necessária para manter a integridade e o acesso às informações em ambiente digital. Era tradicionalmente orientada à implementação de procedimentos de atualização de softwares, de suportes, e de formatos de arquivo, além das rotinas de backup. Conforme sua evolução observou-se uma distinção entre as atividades proferidas sobre as informações, e deste modo, consagraram as estratégias de preservação digital, subdivididas em: estratégias estruturais e operacionais.

As estratégias estruturais se referem aos investimentos iniciais que a organização realiza a fim de viabilizar as ações de preservação digital no longo prazo. Dentre estas estratégias podem ser mencionados: os padrões de metadados, o financiamento da preservação, a adoção de padrões de formatos, os recursos humanos e a infraestrutura tecnológica.

Já as estratégias operacionais se referem às ações proferidas diretamente na informação digital que se deseja preservar. Tais ações impactam diretamente sobre os formatos de arquivo, suportes e equipamentos de hardware e software que são utilizados pelo acervo. Dentre estas estratégias, podem ser mencionados os procedimentos de: migração, emulação, encapsulamento, refrescamento e preservação da tecnologia.

No entanto, a simples implementação de estratégias operacionais configura-se como um ponto crítico no que se refere a autenticidade da informação digital em virtude da vulnerabilidade proporcionada. Portanto, torna-se necessário definir uma sistemática para executar tais intervenções, de modo que seja possível testar os objetos digitais migrados, adicionar metadados, e registrar todas as alterações proferidas no processo de preservação. Em consonância com tal necessidade, surge a implementação de repositórios digitais, para assim, criar um ambiente de preservação no qual as ações possam ser controladas.

Ao transferir as informações digitais para o repositório, tem-se um ambiente complexo para monitorar as atividades e lhes adicionar confiança. Para tanto, a implementação de repositórios digitais deve considerar o modelo Open Archival Information System - OAIS. O modelo OAIS é considerado o principal padrão de referência na preservação digital e tornou-se a norma International Organization for Standardization - ISO 14721:2012. Tal recomendação é ratificada por diversos estudos, dentre eles: Brasil (2015), Day (2004), Fernal e Vechiato (2013), Ferreira (2006), Hedstrom (2001), Márdero Arellano (2004), Santos e Flores (2015), 
Saramago (2004), Sayão (2010), Souza et al. (2012), Thomaz (2006, 2007) e Thomaz e Soares (2004).

Entretanto, o fato dessa temática ser recente atribui dúvidas com relação à confiança depositada pelos usuários potenciais em relação aos métodos utilizados para garantir a preservação, a autenticidade e o acesso. Outrossim, instituições tradicionais de memória, como arquivos, bibliotecas e museus, demoraram um tempo considerável para que pudessem adquirir a confiança desejada. Logo, a informação digital é uma transformação que demanda certo tempo para ser assimilada pelo público. Já aos profissionais da área, delega-se a responsabilidade pela preservação de informações analógicas e digitais.

Mesmo assim, não há metodologias historicamente consagradas para preservação digital, visto que a preocupação com a informação digital é relativamente recente. Portanto, a implementação de repositórios digitais em conformidade com o OAIS busca gerenciar as ações de preservação executadas com a finalidade de demonstrar confiabilidade.

Sendo assim, este estudo tem por objetivo realizar uma reflexão sobre as estratégias de migração no âmbito dos repositórios digitais que seguem o modelo OAIS. Com isso, estima-se descrever os procedimentos necessários para assegurar a autenticidade e o acesso às informações em longo prazo.

O método utilizado consiste no levantamento bibliográfico de materiais previamente publicados, ou seja, parte da seleção de livros e artigos científicos recuperados pela ferramenta de pesquisa Google Scholar; tais materiais pertencem ao recorte temporal entre 1998 e 2018. Utiliza-se o modelo OAIS como prisma da discussão, logo, a implementação das estratégias de migração torna-se a categoria norteadora deste estudo. Os dados coletados são analisados qualitativamente e a discussão segue a lógica dedutiva. Dessa forma, obtém-se um artigo de revisão assistemática que aborda a relação entre o modelo OAIS e as estratégias de migração (GIL, 2010; LUNA, 1997; SILVA; MENEZES, 2005; VOLPATO et al., 2013).

A seguir discute-se, inicialmente, a preservação da informação digital, no que tange à migração de formatos e suportes. E posteriormente, a preservação dos serviços de acesso à informação, por meio do uso de estratégias, considerando as prováveis mudanças tecnológicas do software responsável por garantir o acesso. 


\section{Preservação da informação digital}

A natureza efêmera das mídias de armazenamento aliada às rápidas mudanças da indústria de computadores compõe um antagonismo frente ao propósito fundamental do modelo OAIS: a preservação de informações no longo prazo. Nesse ritmo de evolução acelerada da tecnologia, os sistemas informatizados tornam-se menos rentáveis em virtude da redução de sua perspectiva de longevidade.

Da mesma forma, a manutenção do aparato tecnológico necessário para garantir o acesso aos documentos se tornará mais complexa e isso poderá impactar na informação de representação necessária para preservar a informação de conteúdo (ABNT/NBR 15472:2007; CCSDS, 2012; ISO 14721:2012). Portanto, os objetos digitais não devem ser deixados em formatos obsoletos e somente serem transferidos aos repositórios digitais após um longo período de negligência (MÁRDERO ARELLANO, 2004). É preciso considerar as atividades de preservação desde a produção informacional, de modo que seja possível reunir todos os dados necessários em pacotes de informação para serem preservados em um repositório digital que segue o modelo OAIS.

Com relação ao pacote de informação, pode-se defini-lo como um recipiente conceitual que comporta dois tipos de dados: as informações do conteúdo (content information) e as informações descritivas de preservação (preservation description information - PDI). Assim, informação de conteúdo e PDI são encapsuladas e identificadas por uma informação de empacotamento (packaging information). Como resultado, obtém-se um pacote que pode ser detectado por meio da informação de descrição (descriptive information) inserida (ABNT/NBR 15472:2007; CCSDS, 2012; ISO 14721:2012). A seguir a "Figura 1 - Estrutura do pacote de informação" apresenta os tipos de dados que compõem tal pacote. 
Figura 1 - Estrutura do pacote de informação

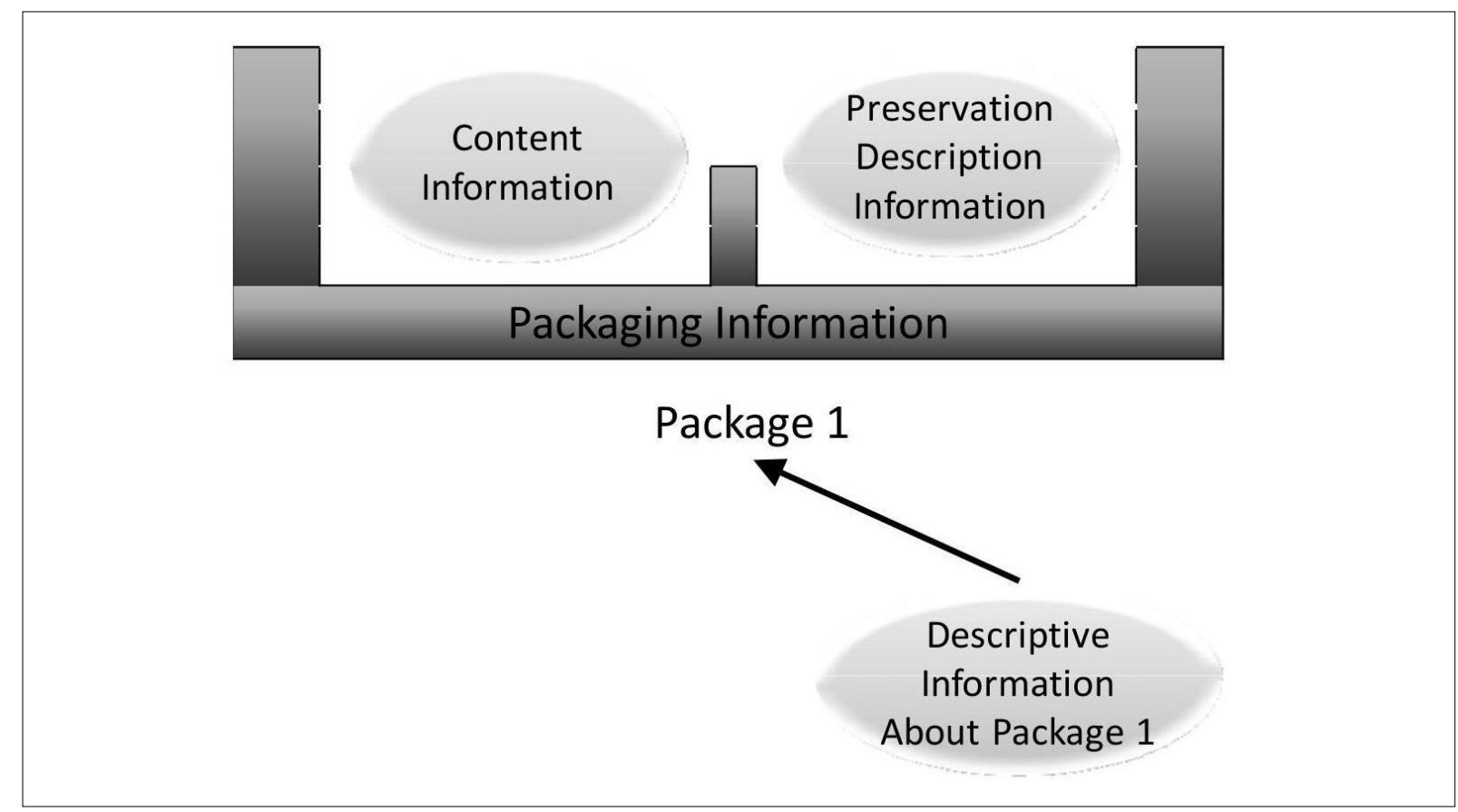

Fonte: CCSDS, 2012, p. 2-6.

Neste pacote genérico, dito Package 1, são identificados a informação de conteúdo (content information), a PDI, a informação de empacotamento (packaging information) e a informação descritiva do "Pacote 1" (descriptive information - About Package 1). A informação de conteúdo (content information) é o conteúdo que se deseja preservar, junto com sua respectiva informação de representação, então necessária para tornar tal conteúdo compreensível à uma determinada comunidade designada. Já a PDI consiste em informações que descrevem procedimentos pertinentes de preservação que são relacionados à procedência, contexto, referência, fixidez e direitos de acesso (ABNT/NBR 15472:2007; CCSDS, 2012; ISO 14721:2012).

A estrutura do pacote de informação é comum às informações que são submetidas, armazenadas e disseminadas pelo repositório OAIS. Sendo assim, para armazenar a informação que se deseja preservar opta-se por um Archival Information Package - AIP, o qual tem por objetivo reunir os componentes digitais necessários para representar corretamente a informação de conteúdo.

Sendo assim, a preservação das informações de conteúdo vislumbra o acesso contínuo em longo prazo para uma comunidade designada, então definida previamente. Deste modo, os pacotes de informação irão se adequar aos conhecimentos de tal comunidade. No entanto, há de se considerar que a base de conhecimento da comunidade designada irá mudar conforme os 
avanços da tecnologia, novamente afetando a informação de representação necessária. Como consequência, a migração de suportes e de plataformas de hardware e software torna-se necessária para que os consumidores tenham acesso às informações digitais.

\section{Estratégias de migração e OAIS}

Para garantir a preservação e o acesso em longo prazo, o repositório digital terá de implementar estratégias de preservação, dentre elas, a mais comum será a migração. Essa estratégia se restringe a atualização de suportes, formatos e versões dos objetos digitais.

A migração consiste na transferência dos dados de uma plataforma de hardware/software potencialmente obsoleta, para uma plataforma atual, tornando os dados compatíveis (GRANGER, 2000; SARAMAGO, 2002). Seu objetivo é prolongar o acesso aos dados por meio da transposição das sequências de bits antigas para tecnologias tidas como contemporâneas.

A migração é a estratégia mais utilizada e compreende atividades de copiar, converter ou transferir, periodicamente, a informação de uma determinada geração de tecnologia para as subsequentes. Dessa forma, é possível copiar a informação de um suporte potencialmente obsoleto ou em estágio de deterioração, para um suporte novo com tecnologia de uso corrente. Além disso, é possível converter os formatos obsoletos para compatibilizá-los com tecnologias atuais, e/ou transferir os objetos digitais armazenados em uma plataforma de hardware/software em processo de obsolescência para outra plataforma de uso corrente. Assim, a migração busca acompanhar a evolução tecnológica, por meio da substituição, logo, torna-se necessário conhecer os suportes de origem e destino (ALMEIDA; CENDÓN; SOUZA, 2012; THOMAZ; SOARES, 2004).

No OAIS, a migração digital consiste na transferência da informação digital que se pretende preservar. Ela se distingue das transferências em geral por três aspectos, que são: o foco na preservação do conteúdo; a nova versão da informação implementada substituirá a anterior; e possuirá controle e responsabilidade total sobre os aspectos da transferência do OAIS (ABNT/NBR 15472:2007; CCSDS, 2012; ISO 14721:2012). 
A migração digital possui uma série de especificidades, que devem ser consideradas no ambiente OAIS. Dentre estas, pode-se enfatizar: as razões para realizar a migração; o contexto da migração e os tipos de migração.

a) Razões para a migração digital: as melhorias do custo-benefício, a necessidade de contemplar novos requisitos para atender ao consumidor, e a substituição da mídia em virtude da sua deterioração;

b) Contexto da migração: corresponde a uma sequência de etapas, iniciando com a identificação da informação de conteúdo. Em seguida, o mapeamento da informação descritiva, a identificação do respectivo AIP. E por fim, a informação de empacotamento irá identificar e delimitar a informação de conteúdo, a PDI, reunindo-as logicamente, em uma única entidade de preservação;

c) Tipos de migração: há quatro tipos de migração compatíveis com o modelo OAIS, que são a renovação, a reprodução, o reempacotamento e a transformação.

A migração deve ser realizada com base em fatores pertinentes a recursos financeiros, necessidade de preservação e perspectivas de acesso. Assim, será preciso identificar e reunir logicamente os componentes do pacote de informação. Dessa forma, podem ser executadas as estratégias de migração conforme as peculiaridades dos objetos digitais. A seguir, os tipos de migração são discutidos a fim de ressaltar vantagens e desvantagens de cada um, o que reforça a necessidade de implementar um conjunto de estratégias.

\section{Renovação}

Envolve estratégias de refrescamento, tendo por finalidade, substituir uma mídia de armazenamento através da cópia exata para uma nova mídia, de modo que, o conjunto de hardware e software da entidade de armazenamento continuará a funcionar como antes (ABNT/NBR 15472:2007; CCSDS, 2012; ISO 14721:2012). A título de exemplo, a renovação ocorre ao se copiar os AIP's armazenados em um Compact Disc - CD antigo (o qual corre risco de deterioração) para um CD novo.

Dessa forma, o conjunto de bits será mantido intacto quando for migrado para novos suportes. Observa-se que este é um estágio crítico da migração, pois expõem o conjunto de bits a possíveis alterações, sejam elas, técnicas ou por demais interesses dos executores do processo 
(INNARELLI, 2009). No entanto, preservar os aspectos físicos, não consiste, essencialmente, em preservar as informações. Logo, a obsolescência tecnológica é uma preocupação significativa que está implícita aos acervos que necessitam da guarda de longo prazo ou permanente (SANTOS, 2005).

O refrescamento por si garante a integridade dos conjuntos de bits, evitando perdas em virtude da deterioração do suporte ou por obsolescência deste. As vulnerabilidades provocadas por essa estratégia, em virtude da exposição dos bits podem ser minimizadas ao realizá-la no ambiente OAIS. Assim, com o auxílio de padrões de metadados é possível registrar as ações proferidas sobre os objetos digitais. Dessa forma, mantém-se um histórico das migrações, capaz de informar a natureza dos suportes que foram perpassados durante o processo de preservação.

\section{Reprodução (replicação)}

Consiste na replicação dos componentes do objeto de informação (informação de empacotamento, informação de conteúdo e PDI) sem causar alterações nos seus respectivos bits. Desta forma, os componentes do objeto de informação podem ser encapsulados e assim, serão facilmente replicados, mantendo a sequência de bits intacta. Este procedimento facilita a migração para novos tipos de mídia de armazenamento e possui baixo risco de perda de informações (ABNT/NBR 15472:2007; CCSDS, 2012; ISO 14721:2012).

O objeto digital pode ser encapsulado junto com o software utilizado para sua criação, e manter ainda, a descrição dos ambientes de software e hardware que são necessários ao seu funcionamento (MÁRDERO ARELLANO, 2004). A correta interpretação desse objeto digital será possível ao reunir todos os componentes digitais necessários. Ao encapsular tais componentes pode-se assegurar uma representação fidedigna da informação, e assim, garantir a autenticidade (FLORES; SANTOS, 2015).

Ao encapsular os objetos digitais, juntamente com os seus respectivos componentes digitais, tem-se um conjunto detalhado de informações necessárias para representar tais objetos. No entanto, essa estratégia visa manter dados intactos durante as migrações de suporte, de modo que, para acessar o conteúdo dos objetos, será preciso implementar outras estratégias como, por exemplo, migração de formato (transformação) ou emulação. 


\section{Reempacotamento}

Tipo de migração necessário quando houver alterações nas informações de empacotamento durante a transferência. Dessa forma, ocorrerá um reempacotamento ao se mover a informação de conteúdo, a informação de empacotamento e a PDI para um novo tipo de suporte. Isto porque os bits usados para representar a informação de empacotamento são alterados (ABNT/NBR 15472:2007; CCSDS, 2012; ISO 14721:2012).

Trata-se de uma espécie de refrescamento semelhante a renovação, entretanto, no reempacotamento ocorre a alteração do tipo de suporte, por exemplo, os AIP's armazenados em um CD são transferidos para um Digital Video Disc - DVD. Consequentemente, os bits da informação de empacotamento são alterados em virtude do novo tipo de suporte.

O refrescamento transfere os objetos digitais fixados em um tipo de suporte, considerado obsoleto, para outro tipo de suporte, de uso corrente, preservando assim, o nível físico do objeto digital. Esse procedimento deve ser realizado antes do suporte obsoleto se deteriorar ou tornese inacessível, em virtude da escassez de periféricos para realizar sua leitura (SANTOS; FLORES, 2017).

Esse tipo de migração também produzirá alterações nos objetos digitais, mesmo que seja apenas nos metadados. Logo, novas informações serão adicionadas, como, por exemplo, a data da migração, a versão do software utilizado para replicação e o tipo do suporte anterior (SANTOS, 2005). Dessa forma, o reempacotamento cria novas informações relacionadas, que são relativas ao procedimento de replicação dos conteúdos de um suporte ao outro. Tais informações devem ser gerenciadas pelo OAIS para agregar confiabilidade às ações de preservação. 


\section{Transformação}

Requer alterações na informação de conteúdo ou na PDI. Logo, as alterações nos bits do objeto de dados se refletem na informação de representação associada que lhe é correspondente. O AIP resultante do processo de transformação irá substituir o AIP que é submetido inicialmente. Assim, o novo AIP é entendido como a versão anterior atualizada. No entanto, observa-se que a primeira versão da AIP (submetida pelo produtor da informação), então dita original, pode ser preservada para fins de verificação da autenticidade das informações preservadas (ABNT/NBR 15472:2007; CCSDS, 2012; ISO 14721:2012).

As transformações podem ser de natureza reversível ou irreversível. Dessa forma, observa-se que a informação de representação desempenha um papel fundamental nas transformações, e os impactos das alterações sobre a mesma podem ser usados para categorizálas.

$\mathrm{Na}$ transformação reversível ocorre a mudança de um determinado padrão por outro semelhante, ressalta-se que haverá equivalência semântica, não ocorrerá perda de informação e por isso será reversível. A compressão sem perdas (lossless compression) de uma imagem digital é um exemplo de transformação reversível.

A compactação será sem perdas caso os dados decodificados sejam idênticos aos dados originais comprimidos pelo codificador (SALOMON, 2004). Da mesma forma, a transformação poderá ser revertida pelo OAIS sem que ocorram perdas de informação.

Já no caso da transformação irreversível não há equivalência semântica, ocorrendo assim, perda de informação, o que impossibilita realizar o caminho reverso. Neste caso, a compressão com perdas (lossy compression) de uma imagem digital é, por exemplo, uma transformação não reversível. Portanto, o OAIS precisa assegurar, para a comunidade designada, que não ocorram perdas significativas de informação.

Certos métodos de compactação com perdas alcançam melhores níveis de compressão. Tal método pode ser aceitável para compactar imagens, áudios ou vídeos caso a perda de dados seja pequena, de modo a apresentar-se imperceptível (SALOMON, 2004). Dessa forma, o OAIS deve definir quais são as propriedades significativas dos objetos digitais que precisam ser preservadas para manter a sua autenticidade. 
É útil preservar as propriedades da informação de transferência como uma propriedade de informação do objeto; logicamente elas não podem verificar se a transformação de natureza irreversível preservou adequadamente a informação de conteúdo, entretanto, podem evidenciar a autenticidade durante este processo de transformação irreversível (CCSDS, 2012; ISO 14721:2012). Observa-se que essa propriedade da informação do objeto atua como um mecanismo que registra as transformações irreversíveis. Dessa forma, tem-se uma visão das transformações ocorridas que corroboram com a presunção de autenticidade.

\section{Migração, propriedades significativas e OAIS: uma possível convergência}

As sucessivas migrações acumulam, progressivamente, alterações na sequência de bits original, assim, podem ocasionar a perda de informações, de funcionalidades, de contexto ou a mudança do objeto conceitual apresentado aos usuários. Tais alterações configuram-se como um problema em potencial no longo prazo, visto que a possibilidade de migrar a versão dos objetos antigos para versões de uso corrente pode ser descontinuada pelo fabricante do software (HEDSTROM, 2001; THIBODEAU, 2002; THOMAZ; SOARES, 2004).

Com a compatibilidade retrospectiva, uma nova geração de softwares poderá interpretar dados de sistemas mais antigos sem necessidade de reformatação ou perda de funcionalidades. Embora tal compatibilidade seja comum nas linhas dos produtos de software, ressalta-se que essas alternativas não são habitualmente fornecidas entre concorrentes, o que poderá dificultar a migração de produtos que foram retirados do mercado de software (HEDSTROM, 1998).

Ao realizar a migração, o repositório deixa de ter a perspectiva do que será exatamente preservado, em virtude da impossibilidade de prever quantas migrações serão necessárias. Portanto, devem-se seguir boas práticas de migração a fim de diminuir o nível de corrupção de dados, além de utilizar técnicas de reparação e registrá-las por meio de metadados (SARAMAGO, 2002). Dessa forma, um repositório que segue o OAIS poderá manter dois objetos digitais (o original e o de preservação) para prevenir quaisquer entraves vindos da descontinuidade dos softwares ou ausência de compatibilidade retrospectiva.

O planejamento da migração requer a definição do formato de arquivo que será usado de modo que possa acomodar as propriedades significativas do objeto digital original; além de definir o aplicativo que irá realizar a migração. Dessa forma, deve-se optar por uma combinação entre o formato de arquivo e o conversor que proporcionem condições de preservar o máximo 
de propriedades do objeto original, considerando o custo mínimo possível (FERREIRA; BAPTISTA; RAMALHO, 2006).

As estratégias de migração variam conforme os softwares utilizados, os conversores e os formatos de arquivo escolhidos como destino da informação que se deseja preservar. Cabe aos conversores replicar as sequências de bits de um formato de arquivo obsoleto para um formato de arquivo de uso corrente, mantendo a maior quantidade possível das propriedades do objeto original. Recai sobre o conversor a responsabilidade de transpor a informação digital através do tempo para mantê-la acessível no longo prazo.

As migrações realizadas pelo OAIS possuem certo grau de especificidade e devem ser executadas com base em uma política de preservação definida a priori. Logo, o repositório deve discernir a necessidade de implementar: renovação, reprodução, reempacotamento ou transformação.

Salienta-se que com exceção da transformação, não será necessário criar uma nova versão do AIP ou mesmo atualizar a sua PDI para os demais casos de migração digital. A versão do AIP possui independência em relação à renovação, reprodução e reempacotamento; visto que tanto a informação de conteúdo, quanto a PDI não são afetadas por estes tipos de migração digital. Mesmo assim, o OAIS verificará se a migração não alterou a informação de conteúdo ou a PDI.

Quando a migração digital envolver transformação será necessário criar uma nova versão do AIP, na qual a PDI necessita ser atualizada para identificar a fonte e a versão do AIP, assim como descrever e justificar as alterações realizadas. Além disso, a descrição associada também necessita ser atualizada. No entanto, isso não implica, necessariamente, em alterar os instrumentos de acesso, salvo casos específicos (ABNT/NBR 15472:2007; CCSDS, 2012; ISO 14721:2012). 


\section{Interface para disseminação das informações}

Com as constantes mudanças tecnológicas, por vezes o OAIS necessita preservar os serviços de acesso aos consumidores. Dessa forma, surge a preocupação com a interface de disseminação, ressaltando questões como o código-fonte do software de acesso e o possível uso das estratégias de emulação para preservar tais serviços.

Haverá casos em que o acesso será mediado por softwares específicos, os quais não são essenciais para preservar as informações do conteúdo, embora sejam convenientes ao acesso. Mesmo assim, esses softwares que apoiam o acesso se tornarão obsoletos com o tempo, e por consequência, deixarão de funcionar em virtude de alterações no ambiente operacional.

Em caráter paliativo, pode-se recriar a funcionalidade desses softwares para um novo ambiente. Tal ação não tem nenhum impacto sobre a preservação da informação de conteúdo, visto que se limita aos softwares de acesso. A recriação dos softwares poderá ter como base, o uso de emuladores, sendo que estes não alteram a informação de conteúdo (ABNT/NBR 15472:2007; CCSDS, 2012; ISO 14721:2012).

A opção do OAIS em preservar um serviço para executar o software de acesso dependerá, essencialmente, da disponibilidade do código-fonte desse aplicativo. Assim, com a posse do código-fonte e da documentação adequada sobre o aplicativo, o procedimento recomendado seria a migração para um novo ambiente, e posteriormente testá-lo para garantir o seu funcionando adequado (ABNT/NBR 15472:2007; CCSDS, 2012; ISO 14721:2012).

A escolha por softwares de padrão aberto reduz o risco de perda da informação digital, especialmente, durante a migração, que é considerada um momento crítico (INNARELLI, 2009). No entanto, a dependência por softwares e formatos de arquivo proprietários, os quais não sejam compatíveis com normas comuns, torna as rotinas de migração frequentes e isoladas (CAMPOS; SARAMAGO, 2007).

Se o software de acesso for um padrão proprietário, amplamente utilizado e disponível comercialmente, é provável que exista um software de conversão usado comercialmente para converter os objetos digitais em outros formatos com aparência semelhante. Observa-se que este tipo de migração, provavelmente será uma transformação de caráter irreversível.

Caso não haja alternativa comercial, o OAIS poderá solicitar uma ferramenta simplificada ao proprietário do software de acesso original, com disponibilidade de parte do 
código-fonte, a fim de acessar as informações. Este procedimento trata-se de uma negociação que envolverá custos adicionais ao processo de preservação, além de manter certo nível de dependência do OAIS em relação ao fabricante do software. Observa-se que essa abordagem pode ser inviável em virtude do custo ou mesmo por questões legais. Em todos os casos, o OAIS deve implementar mecanismos para verificar se nenhuma informação preservada foi perdida; além de garantir a disponibilidade do novo software de acesso à comunidade designada (ABNT/NBR 15472:2007; CCSDS, 2012; ISO 14721:2012).

Para evitar a dependência por padrões proprietários, a informação digital precisa ser produzida por software livre e registrada em formatos de arquivo abertos. Com isso, podem-se escolher padrões de preservação, amplamente difundidos, sem compressão (ou de compressão sem perdas). Portanto, as atividades de preservação digital devem ser iniciadas antes mesmo da produção da informação, visando diminuir os gastos tanto com licenças de softwares, quanto com a execução de estratégias de migração (SANTOS; FLORES, 2018).

Alternativamente à migração, as estratégias de emulação podem ser utilizadas quando um determinado sistema operacional deve ser executado em uma plataforma de hardware para a qual não foi projetado. Assim, o emulador deverá reproduzir a plataforma para possibilitar o correto processamento dos dados, tendo em vista a sua acepção “original”.

A emulação de um ambiente operacional com poucas aplicações a serem suportadas irá reduzir o risco de perda de informação, tornando sua implementação viável para o OAIS. Desta forma, a emulação poderá apoiar a preservação de longo prazo, sendo importante destacar que ela provoca alterações na PDI associada ao AIP, necessitando ser documentada; logo, é considerada como um tipo de migração digital, mais precisamente, uma transformação (CCSDS, 2012; ISO 14721:2012).

Entretanto, alguns recursos específicos podem ser perdidos em virtude da falta de compatibilidade. Por essa razão, há investigações que abordam formas alternativas de emulação, como o desenvolvimento de máquinas virtuais ou emulação em nível de sistema operacional. Tais abordagens resolvem alguns dos problemas de emulação de hardware, porém introduzem complexidade e consequentemente, novas preocupações (ABNT/NBR 15472:2007; CCSDS, 2012; ISO 14721:2012).

Tal anteposição é típica da natureza recursiva da informação digital, pois para preservála será necessário adicionar mais informações digitais, e assim sucessivamente. Tal expansão 
de complexidade pode ser minimizada ao relacionar e descrever a informação por meio de formatos de simples compreensão, até mesmo existentes em meio analógicos, como por exemplo, os códigos American Standard Code for Information Interchange - ASCII ou The Unicode Standard - UNICODE.

\section{Considerações finais}

Este estudo perpassou aspectos da preservação digital, dentre eles: a implementação de repositórios digitais em conformidade com o modelo OAIS; o uso das estratégias de migração de formatos e suportes; e a preservação dos serviços de acesso à informação digital. Para isso, partiu-se de uma revisão assistemática capaz de refletir sobre a convergência entre o OAIS e as estratégias de migração.

Inicialmente, apresentou-se um breve panorama da preservação digital enfatizando a fragilidade implícita da informação registrada em ambiente digital. Logo, observou-se a necessidade de implementar repositórios digitais em conformidade com o modelo OAIS para garantir a preservação de longo prazo. Por conseguinte, a estrutura do pacote de informação foi detalhada para elucidar a implementação de estratégias de migração pelo OAIS.

Tais estratégias tornam-se necessárias para manter o acesso a plataformas obsoletas por meio de tecnologias de uso corrente. Dentre estas estratégias, variantes da migração, destacamse: renovação, reprodução, reempacotamento e transformação. Ressalta-se que as estratégias devem ser implementadas no ambiente OAIS, a fim de aumentar os níveis de confiabilidade do processo de preservação.

Dentre as variantes da migração, observa-se que a renovação se limita a efetuar o refrescamento do suporte, evitando assim, a sua deterioração e consequente perda das informações. Já a reprodução se concentra em encapsular todos os componentes em um pacote de informação, mantendo os dados intactos. Observa-se que tal variação pode ser complementada no futuro, com a migração de formato ou emulação.

Posteriormente, o reempacotamento é muito similar a renovação, entretanto, este consiste em mudança da natureza do suporte, de modo que as sequências de bits serão reorganizadas no novo suporte. Já a transformação, pode ser subdividida em duas categorias: reversível e irreversível. Na transformação reversível há equivalência semântica, de modo a 
permitir que o OAIS realize a reversão. Já na transformação irreversível não há tal equivalência, de modo que ocorre a perda de informações. Logo, na transformação irreversível, deve-se atentar para a definição das propriedades significativas.

Da mesma forma, observa-se que sucessivas migrações podem ocasionar perdas de informações, fato que poderá ser agravado com a impossibilidade de migrar os formatos caso ocorra uma possível incompatibilidade futura entre as versões dos softwares. Tal entrave reforça a necessidade de um planejamento da preservação no qual serão definidos os formatos de arquivo e os suportes que serão utilizados, com objetivo de evitar a dependência.

Em caráter complementar à preservação, o OAIS pode fornecer serviços de acesso à informação, mediados por softwares, com o objetivo de facilitá-los. Entretanto, assim como qualquer outro software, poderá tornar-se obsoleto, fazendo necessária a implementação de estratégias de emulação para manter as suas funcionalidades.

Recai aqui a importância dos padrões abertos, de modo que podem reduzir os esforços de preservação e evitar a dependência tecnológica, especialmente de padrões proprietários. Para isso, ressalta-se a proeminência de que a informação digital seja produzida e registrada por softwares e formatos de arquivo abertos. Tal requisito também pode ser ressaltado pelo OAIS no momento que o produtor realiza a submissão dos pacotes de informação.

Por fim, este estudo contribui para clarificar a relação entre modelo OAIS e as estratégias de migração, demonstrando a aplicabilidade de tipos comuns de migração. Discutiuse ainda, os serviços de acesso que o OAIS pode oferecer a sua comunidade designada. Pondera-se que o OAIS é o ambiente confiável para proferir as estratégias e registrá-las, a fim de manter a autenticidade da informação. Portanto, estima-se incentivar o diálogo em torno dos repositórios digitais, de modo que esta reflexão forneça subsídios teóricos aos pesquisadores recém-iniciados na temática. A principal limitação deve-se a sua natureza puramente teórica, sendo assim, recomenda-se o desenvolvimento de estudos de caso para ampliar a discussão sobre o tema. 


\section{Referências}

ASSOCIAÇÃO BRASILEIRA DE NORMAS TÉCNICAS (ABNT). NBR 15472: sistemas espaciais de dados e informações: modelo de referência para um sistema aberto de arquivamento de informação (SAAI). Rio de Janeiro: ABNT, 2007.

ALMEIDA, M. B.; CENDÓN, B. V.; SOUZA, R. R. Metodologia para implantação de programas de preservação de documentos digitais a longo prazo. Encontros Bibli: revista eletrônica de Biblioteconomia e Ciência da Informação, v. 17, n. 34, p. 103-130, maio/ago., 2012. Disponível em: https://doi.org/10.5007/1518-2924.2012v17n34p103. Acesso em: 20 dez. 2018.

BRASIL. Conselho Nacional de Arquivos. Câmara Técnica de documentos eletrônicos. Diretrizes para a implementação de repositórios arquivísticos digitais confiáveis - RDCArq. Rio de Janeiro: Arquivo Nacional, 2015. Disponível em: http://www.conarq.gov.br/images/publicacoes_textos/diretrizes_rdc_arq.pdf. Acesso em: 10 jun. 2016.

CAMPOS, F. M. G.; SARAMAGO, M. L. Preservação digital de longo prazo em instituições patrimoniais: reutilização e adaptação de metadados. Actas dos Congressos Nacionais de Bibliotecários, Arquivistas e Documentalistas, Lisboa, n. 9, 2007. Disponível em: http://www.bad.pt/publicacoes/index.php/congressosbad/article/view/540/330. Acesso em: 09 jul. 2018.

CONSULTATIVE COMMITTEE FOR SPACE DATA SYSTEM (CCSDS). Reference model for an Open Archival Information System (OAIS): magenta book. Washington: CCSDS, 2012. Disponível em: http://public.ccsds.org/publications/archive/650x0m2.pdf. Acesso em: 13 maio 2014.

DAY, M. Preservation metadata. In: GORMAN, G. E.; DORNER, D. G. (ed.) Metadata applications and management: International Yearbook of Library and Information Management. London: Facet, 2004. p. 253-273. Disponível em: https://researchportal.bath.ac.uk/en/publications/preservation-metadata. Acesso em: 09 dez. 2018.

FERNAL, A.; VECHIATO, F. L. Repositórios digitais como ambientes de atuação do arquivista: um estudo dos princípios arquivísticos e da preservação digital nesse contexto. Informação@Profissões, Londrina, v. 2, n. 1, p. 103-122, 2013. Disponível em: http://www.uel.br/revistas/uel/index.php/infoprof/article/view/17272. Acesso em: 10 jan. 2016.

FERREIRA, M. Introdução à preservação digital: conceitos, estratégias e actuais consensos. Portugal: Escola de Engenharia da Universidade do Minho, 2006. Disponível em: https://repositorium.sdum.uminho.pt/bitstream/1822/5820/1/livro.pdf. Acesso em: 02 ago. 2014.

FERREIRA, M.; BAPTISTA, A. A.; RAMALHO, J. C. A foundation for automatic digital preservation. Ariadne: web magazine for information professionals, 2006. Disponível em: http://www.ariadne.ac.uk/issue/48/ferreira-et-al/. Acesso em: 10 jan. 2015. 
FLORES, D.; SANTOS, H. M. Preservação de documentos arquivísticos digitais: reflexões sobre as estratégias de encapsulamento. Liinc em Revista, Rio de Janeiro, v. 11, n. 1, p. 167 180, maio 2015. Disponível em: https://doi.org/10.18617/liinc.v11i1.770. Acesso em: 02 jul. 2015.

GIL, A. C. Como elaborar projetos de pesquisa. 4. ed. São Paulo: Atlas, 2010.

GRANGER, S. Emulation as a digital preservation strategy. D-Lib Magazine, v. 6, n. 10, 2000. Disponível em: http://www.dlib.org/dlib/october00/granger/10granger.html. Acesso em: 24 jun. 2015.

HEDSTROM, M. Digital preservation: a time bomb for digital libraries. Computer and the humanities, Netherlands, n. 31, p. 189-202, 1998. Disponível em:

http://deepblue.lib.umich.edu/bitstream/2027.42/42573/1/10579_2004_Article_153071.pdf.

Acesso em: 01 out. 2014.

HEDSTROM, M. Digital preservation: problems and prospects. Michigan: University of Michigan, 2001. Disponível em: http://www.dl.slis.tsukuba.ac.jp/DLjournal/No_20/1hedstrom/1-hedstrom.html. Acesso em: 10 jan. 2019.

INNARELLI, H. C. Preservação digital e seus dez mandamentos. In: SANTOS, V. B. (org.). Arquivística: temas contemporâneos, classificação, preservação digital, gestão do conhecimento. 3. ed. Brasília: SENAC, 2009. p. 21-75.

INTERNATIONAL ORGANIZATION FOR STANDARDIZATION. ISO 14721: space data and information transfer systems: open archival information system: reference model. Genebra: ISO, 2012.

LUNA, S. V. Planejamento de pesquisa: uma introdução. São Paulo: EDUC, 1997.

MÁRDERO ARELlAnO, M. Á. Preservação de documentos digitais, Ciência da Informação, Brasília, v. 33, n. 2, p. 15-27, maio/ago. 2004. Disponível em: http://revista.ibict.br/ciinf/article/view/1043. Acesso em: 25 jul. 2014.

SALOMON, D. Data compression: the complete reference. 3. ed. New York: Springer, 2004.

SANTOS, V. B. Gestão de documentos eletrônicos: uma visão arquivística. 2. ed. Brasília: Abarq, 2005.

SANTOS, H. M.; FLORES, D. Preservação de documentos arquivísticos digitais: reflexões sobre o uso de padrões abertos nos acervos. Investigación Bibliotecológica: archivonomía, bibliotecología e información, Cidade do México, v. 32, n. 74, p. 35-53, 2018. Disponível em: http://dx.doi.org/10.22201/iibi.24488321xe.2018.74.57905. Acesso em: 11 dez. 2018.

SANTOS, H. M.; FLORES, D. Preservação de documentos digitais: reflexões sobre as estratégias de refrescamento. RBBD: revista brasileira de Biblioteconomia e Documentação, São Paulo, v. 13, n. 2, p. 31-41, 2017. Disponível em: https://rbbd.febab.org.br/rbbd/article/view/449. Acesso em: 23 jan. 2019. 
SANTOS, H. M.; FLORES, D. Repositórios digitais confiáveis para documentos arquivísticos: ponderações sobre a preservação em longo prazo. Perspectivas em Ciência da Informação, Belo Horizonte, v. 20, n. 2, p. 198-218, abr./jun. 2015. Disponível em: http://dx.doi.org/10.1590/1981-5344/2341. Acesso em: 03 dez. 2018.

SARAMAGO, M. L. Metadados para preservação digital e aplicação do modelo OAIS. In: CONGRESSO NACIONAL DE BIBLIOTECÁRIOS, ARQUIVISTAS E DOCUMENTALISTAS, 8., 2004. Anais eletrônicos... Estoril: [s. n.], 2004. Disponível em: http://www.bad.pt/publicacoes/index.php/congressosbad/article/view/640/637. Acesso em: 04 jul. 2014.

SARAMAGO, M. L. Preservação digital a longo prazo: boas práticas e estratégias. Cadernos BAD: Revista da Associação Portuguesa de Bibliotecários, Arquivistas e Documentalistas, Lisboa, n. 2, p. 54-68, 2002. Disponível em:

http://www.bad.pt/publicacoes/index.php/cadernos/article/view/866. Acesso em: $10 \mathrm{fev}$. 2015.

SAYÃO, L. F. Repositórios digitais confiáveis para a preservação de periódicos eletrônicos científicos. Ponto de Acesso, Salvador, v. 4, n. 3, p. 68-94, dez. 2010. Disponível em: https://portalseer.ufba.br/index.php/revistaici/article/view/4709/3565. Acesso em: 08 ago. 2018.

SILVA, E. L.; MENEZES, E. M. Metodologia da pesquisa e elaboração de dissertação. 4. ed. rev. atual. Florianópolis: UFSC, 2005. Disponível em:

https://www.researchgate.net/publication/312125489. Acesso em: 16 dez. 2018.

SOUZA, A. H. L. R.; OLIVEIRA, A. F.; D’AVILA, R. T.; CHAVES, E. S. S. O modelo de referência OAIS e a preservação digital distribuída. Ciência da Informação, Brasília, v. 41, n. 1, p. 65-73, jan./abr., 2012. Disponível em: http://revista.ibict.br/ciinf/article/view/1352. Acesso em: 18 mar. 2015.

THIBODEAU, K. Overview of technological approaches to digital preservation and challenges in coming years. In: COUNCIL ON LIBRARY AND INFORMATION RESOURCES. The state of digital preservation: an international perspective. Washington, 2002. p. 4-31. Disponível em:

http://citeseerx.ist.psu.edu/viewdoc/summary?doi=10.1.1.89.3273. Acesso em: 19 ago. 2014.

THOMAZ, K. P. Gestão e preservação de documentos eletrônicos de arquivo: revisão de literatura - parte 2. Arquivística.net, Rio de Janeiro, v. 2, n. 1, p. 114-131, jan./jun. 2006. Disponível em:

http://www.brapci.inf.br/index.php/article/view/0000003988/bb2cabd823d3068f4d817793e6a 11b68. Acesso em: 07 set. 2014.

THOMAZ, K. P. Repositórios digitais confiáveis e certificação. Arquivística.net, Rio de janeiro, v. 3, n. 1, p. 80-89, jan./jun. 2007. Disponível em:

http://www.brapci.inf.br/_repositorio/2010/05/pdf_fed0720dbb_0010726.pdf. Acesso em: 07 set. 2014. 
THOMAZ, K. P.; SOARES, A. J. A preservação digital e o modelo de referência Open Archival Information System (OAIS). DataGramaZero, Rio de Janeiro, v. 5, n. 1, 2004. Disponível em: http://www.brapci.inf.br/index.php/article/download/7616. Acesso em: 10 dez. 2017.

VOLPATO, G. L.; BARRETO, R. E.; UENO, H. M.; VOLPATO, E. D. S. N.; GIAQUINTO, P. C.; FREITAS, E. G. D. Dicionário crítico para redação científica. Botucatu: Best Writing, 2013. 